\title{
Completely Asynchronous Optimistic Recovery with Minimal Rollbacks
}

\author{
Sean W. Smith ${ }^{*}$ David B. Johnson, and J. D. Tygar \\ Computer Science Department \\ Carnegie Mellon University \\ Pittsburgh, PA 15213-3891 USA
}

\begin{abstract}
Consider the problem of transparently recovering an asynchronous distributed computation when one or more processes fail. Basing rollback recovery on optimistic message logging and replay is desirable for several reasons, including not requiring synchronization between processes during failure-free operation. However, previous optimistic rollback recovery protocols either have required synchronization during recovery, or have permitted a failure at one process to potentially trigger an exponential number of process rollbacks. In this paper, we present an optimistic rollback recovery protocol that provides completely asynchronous recovery, while also reducing the number of times a process must roll back in response to a failure to at most one. This protocol is based on comparing timestamp vectors across multiple levels of partial order time.
\end{abstract}

\section{Introduction}

\subsection{The Problem}

Consider a long-running application program on an asynchronous distributed system. If a process $p$ fails and then recovers by rolling back to a previous state, process $p$ 's computation since it first passed through the restored state has become lost. The failure and rollback of $p$ may cause the state at a surviving process to become an orphan - a state that causally depends on lost computation - and the existence of orphan states causes the system state to be inconsistent. The challenge of rollback recovery is to restore and maintain a consistent system state when one or more processes fail and roll back.

This research was sponsored in part by the Advanced Research Projects Agency under contract numbers F19628-93-C-0193 and DABT63-93-C-9954, IBM, Motorola, the National Science Foundation under Presidential Young Investigator Grant CCR-8858087, TRW, and the U.S. Postal Service. The first author also received support from an ONR Graduate Fellowship. The views and conclusions contained in this document are those of the authors and should not be interpreted as representing the official policies, either expressed or implied, of the U.S. Government.

${ }^{*}$ Dr. Smith is currently affiliated with the Computer Research and Applications group at Los Alamos National Laboratory, Mail Stop M986, P.O. Box 1663, Los Alamos, NM 87545 USA.
One desirable property of a rollback recovery protocol is the ability to perform recovery transparently to the application program. Another desirable property is minimizing the amount of computation wasted due to rollback, such as the number of surviving processes that must roll back, the number of times each process must roll back, or the amount of rolled-back computation beyond that which causally depends on the computation lost due the failure; an extreme case of such wasted computation is the domino effect [22, 24]. A third desirable property is minimizing the overhead incurred by the protocol, both during failure-free execution and during recovery. During failure-free execution, synchronization between processes slows down the application program; during recovery, synchronization between processes slows down recovery and may prevent recovery from concurrent failures from proceeding concurrently.

Optimally, surviving processes roll back at most once, and only roll back the portion of their own computation that has become an orphan. In this paper, we present a protocol that provides this property, while also providing complete asynchrony. Processes do not synchronize with each other during failure-free execution or during recovery, and processes record all recovery information during failure-free operation (logged messages and checkpoints) on stable storage asynchronously.

\subsection{Optimistic Message Logging}

Our protocol is based on optimistic message logging.

In the general message logging approach to recovery, processes $\log$ their received messages and occasionally checkpoint their local state. A process may recover to any past state by restarting from an earlier checkpoint and then replaying from the log the sequence of messages it originally received after that checkpoint. Message logging assumes that the execution of each process is piecewise deterministic $^{1}$ [30] This scheme can also be extended to handle some

\footnotetext{
${ }^{1}$ That is, execution between successive received messages is completely determined by the process state before the first of these messages is received and by contents of that message. After receiving a message, a process performs a sequence of deterministic state transitions, some of which may involve sending messages to other processes. The process then attempts to receive another message, and blocks until one is available.
} 
nondeterminism $[9,14]$ by treating each nondeterministic influence as a message, logging it and replaying it during recovery.

The message logging approach allows states of a process in addition to those saved in a checkpoint to be recovered. Recovery protocols based instead on checkpointing without message logging (e.g., $[1,5,6,7,8,15,16,17,31])$ can recover only process states that have been checkpointed, often forcing processes to roll back further than otherwise required after a failure. Message logging allows each process to be checkpointed less frequently, and may in general reduce failure-free overhead since logging a message is less expensive than recording a checkpoint. Message logging also avoids the need for process synchronization during checkpointing.

Optimistic message logging protocols (e.g., [12, 13, 14, $20,25,30]$ ) buffer received messages in volatile storage and log them to stable storage asynchronously in order to avoid blocking the process due to logging. Unlike pessimistic message logging protocols (e.g., $[3,4,9,11,21]$ ), optimistic protocols allow a process to receive a message and to continue execution before the message is logged to stable storage. Since a failed process that has not yet logged some received messages may not be able to restore its last state before failure, this failure may cause other processes to become orphans. In order to restore the system to a consistent state, an optimistic recovery protocol must be able to detect and eliminate orphans throughout the system. Although optimism thus complicates recovery, optimistic rollback protocols are potentially cheaper during failurefree operation due to their asynchronous operation. By also using asynchronous checkpointing techniques [8, 17], all causes of process blocking due to fault tolerance during failure-free operation may be avoided.

\subsection{Asynchronous Recovery}

Existing optimistic rollback protocols have required synchronization between processes during recovery, in order to ensure that the system recovers to a consistent state and that the system state remains consistent in spite of the effects of any orphan processes or messages remaining after the failure.

Strom and Yemini [30] initiated the area of optimistic rollback recovery and presented the most asynchronous protocol prior to the completely asynchronous protocol that we present in this paper. In the Strom and Yemini protocol, processes use timestamp vectors to track dependency. When a process rolls back, it begins a new incarnation and sends announcements to the other processes. (These announcement messages are not part of the failure-free computation, and thus do not carry dependency.) When a process receives a rollback announcement, it uses its timestamp vector to determine if its current state is an an orphan; if so, this process rolls back to its maximal state that it believes is not an orphan: the process restarts from an old checkpoint and replays from the log its received messages until it reaches one known to be an orphan based on the incarnation start information contained in the rollback announcements it has received.

The Strom and Yemini protocol usually requires no process synchronization during recovery, but may in some cases need to block a process. Processes need the incarnation start information from rollback announcements in order to compare timestamp vectors received on messages. (Otherwise, when a message from a new incarnation arrives, a process does not know whether to accept and continue, or to roll back.) If an announcement is delayed, any process that has not yet received the announcement may be forced to block when it needs to make a timestamp vector comparison. This behavior can occur even though the protocol assumes FIFO message ordering between each pair of processes, since a timestamp vector entry referring to the new incarnation may arrive at this process indirectly through a chain of messages.

In addition, the asynchrony present in the Strom and Yemini protocol can permit a single failure at one process to cause other processes to roll back an exponential number of times. This behavior occurs because an orphan state at a surviving process $r$ may depend on the lost computation at another process through multiple paths: directly from the failed process, and indirectly through intermediate processes. The protocol may generate rollback announcements in such a way that process $r$ rolls back in response to the rollbacks of intermediate processes, and then in response to the rollback of the failed process. Figure 1 shows a simple scenario in which process $r$ rolls back twice in response to a single failure at process $p$. Sistla and Welch [25] claim an upper bound of $O\left(2^{n}\right)$ rollbacks in the worst case for the Strom and Yemini protocol, where $n$ is the number of processes in the system. In [27], we construct an explicit example showing $2^{n}-1$ rollbacks, and thus establish a tight bound $\Theta\left(2^{n}\right)$ for the worst case.

\subsection{Our Results}

In this paper, we present a new protocol for optimistic rollback recovery. Much previous work in optimistic recovery has modeled the application program with partial order time, and used the standard technique of timestamp vectors $[10$, $18,30]$ to track causal dependency. Our work exploits the insight that the transparent recovery protocol itself is also an asynchronous distributed computation. This recovery computation can also modeled by a partial order - but one that differs from the partial order for the user application computation. Our protocol maintains vector clocks for both levels; comparing vectors across time levels optimally characterizes when a given state is an orphan. 


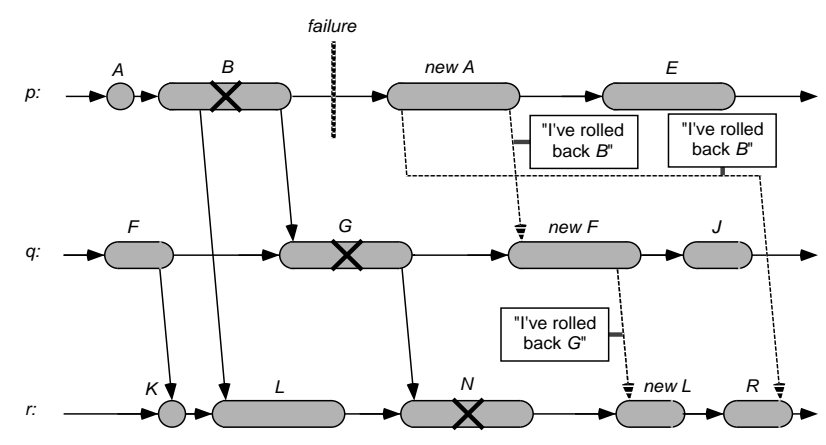

Figure 1 In this example of how the Strom and Yemini protocol may cause surviving processes to roll back multiple times in response to a single failure, one failure at process $p$ causes process $r$ to roll back twice. Process $p$ fails and rolls back state interval $B$, making state intervals $G$ and $N$ orphans. When $q$ receives $p$ 's announcement about $B, q$ rolls back to its most recent state that does not depend on $B$, but $q$ 's announcement may arrive at $r$ before $p$ 's announcement does. Process $r$ does not know that its restored state is still an orphan until after $p$ announcement also arrives. (An " $\mathrm{X}$ " marks each rolled-back interval.)

Our new protocol improves on previous optimistic rollback protocols by requiring no synchronization during recovery. In comparison specifically to the Strom and Yemini protocol (which otherwise requires the least synchronization during recovery), our protocol reduces the worst case number of rollbacks per process after any failure from exponential to one, and requires neither FIFO messages nor blocking of any process. Our protocol also does not require the sending of any messages other than those sent by the application program. The principal drawback of our protocol is timestamp size, since the protocol requires vector clocks for two levels of partial order time. Table 1 compares our protocol to four principal optimistic message logging protocols (discussed further in Section 5).

\section{Theoretical Basis}

\subsection{Definitions}

Two Levels of Computation Besides performing the application program, processes also perform recovery. We formalize this duality by discussing two distributed computations:

- the user application computation, and

- the system recovery computation.

The system process at $p$ implements the user process (but the system process is transparent to the user process). The system state at process $p$ consists of the user state plus some extra state. All user messages are carried by system

\begin{tabular}{|c|c|c|c|c|c|}
\hline & 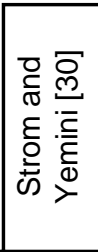 & 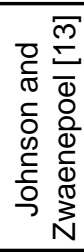 & 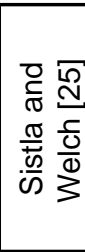 & 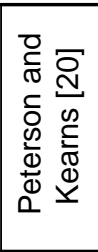 & 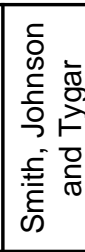 \\
\hline Assumptions & FIFO & None & FIFO & None & None \\
\hline $\begin{array}{l}\text { Asynchronous } \\
\text { recovery }\end{array}$ & Mostly & No & No & No & Yes \\
\hline $\begin{array}{l}\text { Worst case } \\
\text { rollbacks at one } \\
\text { process from } \\
\text { one failure }\end{array}$ & $\Theta\left(2^{n}\right)$ & 1 & 1 & 1 & 1 \\
\hline $\begin{array}{l}\text { Entries in } \\
\text { timestamps }\end{array}$ & $\mathrm{O}(\mathrm{n})$ & $\mathrm{O}(1)$ & $O(n)$ & $O(n)$ & $\mathrm{O}(\mathrm{n})$ \\
\hline
\end{tabular}

Table 1 Summary of comparison to previous work.

messages, but some messages may be exclusively systemonly. Only user messages are logged.

State Intervals A state interval is a period of deterministic execution at a process. In our model, each process has a current system state interval and a current user state interval. A process begins a new system state interval at each occurrence of any of three events: the system process receives a new system-level message; the user process receives a new user-level message; or the system process rolls back the user process. A process begins a new user state interval each time the user process receives a new user-level message. Rollback restores an old user state interval.

Each state interval at a process has a state interval index. We use capital Roman letters to denote state interval indices, and use subscripts to indicate whether the interval is from the system level or from the user level. For example, $A_{S}$ denotes a system state interval index, and $B_{U}$ denotes a user state interval index.

At each process, the system state interval indices form a timeline: a linear sequence. The user state interval indices, on the other hand, form a timetree, since each rollback begins a new branch. Figure 2 illustrates this branching. We use $\preceq_{S}$ and $\preceq_{U}$ to indicate comparison on system state interval indices and user state interval indices, respectively. (These comparisons are valid only for intervals within a single process.)

Relating System State to User State Since (at each process) a new system state interval begins whenever a new user state interval begins, a given system state interval is associated with exactly one user state interval.

We can use this association to compare system state interval indices to user state interval indices. We denote this 


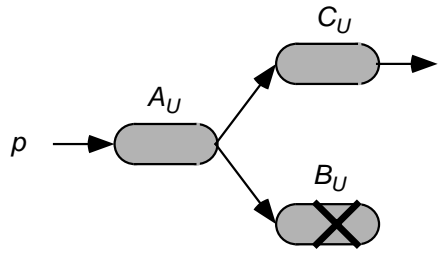

Figure 2 Rollback places the user state interval indices at a process into a timetree. Here, process $p$ executes user state interval $A_{U}$, then $B_{U}$, then rolls back to $A_{U}$, then executes $C_{U}$.

comparison by $\preceq_{*}$. For any state interval indices $A_{U}$ and $B_{S}$, we define $A_{U} \preceq_{*} B_{S}$ to hold if and only if $A_{U} \preceq_{U} B_{U}$, where $B_{U}$ is the user state interval index associated with $B_{S}$.

We also introduce functions to make this association explicit. Let SYS_TO_USR map each system state interval to its user state interval, and let $U S R_{-} T O_{-} S Y S$ map each user state interval to its set of system state intervals.

Vectors of Indices A vector is an array of state interval indices, one for each process. For a vector $X, X[p]$ denotes the process $p$ entry of $X$. We use the $\preceq_{S}, \preceq_{U}$, and $\preceq_{*}$ comparisons on state interval indices to define entrywise comparisons on vectors of state interval indices. We also use the $\preceq_{S}$ and $\preceq_{U}$ comparisons to define the entrywise vector maximizations $M A X_{S}$ and $M A X_{U}$, respectively. However, since user state interval indices form a tree, it is possible that two user state interval indices at a process may be incomparable. (For example, in Figure 2, $B_{U}$ and $C_{U}$ are incomparable.) $M A X_{U}$ is undefined for such inputs.

Rollback Suppose user state interval $A_{U}$ and system state interval $B_{S}$ both occur at process $p$. We say that $B_{S}$ rolls back $A_{U}$ when $B_{S}$ is the state interval that process $p$ started when it began rolling back during recovery, and $A_{U}$ was one of the user state intervals rolled back. Since process $p$ can only roll back something that has already happened, any $A_{S} \in U S R_{-} T O_{-} S Y S\left(A_{U}\right)$ satisfies $A_{S} \preceq_{S} B_{S}$.

Two Partial Orders We build abstract partial order time models for the two levels of computation in our system. We obtain these partial orders by constructing directed acyclic graphs whose nodes represent state interval indices and whose edges represent precedence.

For the system computation, we construct a node for each system state interval index. Let $A_{S}$ and $B_{S}$ be distinct state interval indices. We draw an edge from $A_{S}$ to $B_{S}$ when $A_{S} \preceq_{S} B_{S}$, or when $A_{S}$ sends a message that initiates $B_{S}$. We say that $A_{S}$ precedes $B_{S}$ in the system partial order when a path exists from $A_{S}$ to $B_{S}$ in this graph. For the user computation, we construct a node for each user state interval index. Let $A_{U}$ and $B_{U}$ be distinct state interval indices. We draw an edge from $A_{U}$ to $B_{U}$ when $A_{U} \preceq_{U} B_{U}$, or when
$A_{U}$ sends a message that initiates $B_{U}$. We say that $A_{U}$ precedes $B_{U}$ in the user partial order when a path exists from $A_{U}$ to $B_{U}$ in this graph. We use $\rightarrow$ to indicate precedence in these partial orders, and $\longrightarrow$ to indicate precedence or equality. Which partial order should be clear from the subscripts on the variable names. (Note that unlike the $\preceq_{U}$ and $\preceq_{S}$ relations, these partial orders may relate intervals at different processes.)

The fact that rollback places the user state interval indices at a process into a tree permits some pathological situations. To restrict these situations, we say that a user state interval $A_{U}$ is valid when its past in the user partial order touches only one root-leaf path in the timetree at any process. For example, consider Figure 2; any user state interval $D_{U}$ (at any process) that satisfied $B_{U} \rightarrow D_{U}$ and $C_{U} \rightarrow D_{U}$ would not be valid.

Timestamp Vectors Timestamp vectors $[10,18]$ are a well-known technique for tracking partial order time relations. The timestamp vector for a state interval $A$ consists of, for each process $q$, the maximal interval at $q$ that precedes or equals $A$ in the partial order. Comparing the timestamp vectors for two state interval indices determines their partial order relation.

This property applies to both partial orders we construct. Suppose system state intervals $A_{S}$ and $B_{S}$ have timestamp vectors $X_{S}$ and $Y_{S}$, respectively. Then $A_{S} \longrightarrow B_{S}$ if and only if $X_{S} \prec_{S} Y_{S}$. Similarly, suppose valid user state intervals $A_{U}$ and $B_{U}$ have timestamp vectors $X_{U}$ and $Y_{U}$, respectively. Then $A_{U} \longrightarrow B_{U}$ if and only if $X_{U} \preceq_{U} Y_{U}$.

In particular, if $X_{S}$ is the timestamp vector for system state interval $B_{S}$, then a system state interval $A_{S}$ at a process $p$ satisfies $A_{S} \longrightarrow B_{S}$ if and only if $A_{S} \preceq_{S} X_{S}[p]$. Similarly, if $X_{U}$ is the timestamp vector for valid user state interval $B_{U}$, then a user state interval $A_{U}$ at a process $p$ satisfies $A_{U} \longrightarrow B_{U}$ if and only if $A_{U} \preceq_{U} X_{U}[p]$.

\subsection{The Orphan Comparison}

Potential Knowledge Suppose $A_{U}$ is a user state interval at process $p$. When can another process potentially be aware that $A_{U}$ is an orphan?

We define a predicate KNOWABLE_ORPHAN $\left(A_{U}, B_{S}\right)$ that is true when $A_{U}$ is an orphan or has been rolled back, and process $q$ during system state interval $B_{S}$ can potentially be aware of this fact. For the predicate to be defined, process $q$ must be aware of $A_{U}$; hence we require the precondition that $A_{S} \longrightarrow B_{S}$ for some $A_{S} \in U S R_{-} T O_{-} S Y S\left(A_{U}\right)$. For the predicate to be true, there must exist a user state interval $C_{U}$ and a system state interval $D_{S}$ satisfying three conditions: first, $C_{U} \longrightarrow A_{U}$; second, $D_{S}$ rolls back $C_{U}$; and third, $D_{S} \Longrightarrow B_{S}$. The first two conditions are necessary for $A_{U}$ to no longer be part of the valid computation. The last condition is necessary for process $q$ to be potentially aware of this fact at $B_{S}$. 
An Optimal Test Comparing timestamp vectors across the two levels of partial order time exactly captures this potential knowledge.

Theorem 1 Suppose user state interval $A_{U}$ at process $p$ and system state interval $B_{S}$ at process $q$ satisfy $A_{S} \longrightarrow B_{S}$, for some $A_{S}$ in $U S R_{-} T O_{-} S Y S\left(A_{U}\right)$. Let $X_{U}$ be the user timestamp vector of $A_{U}$, and let $Y_{S}$ be the system timestamp vector of $B_{S}$. Then $K N O W A B L E_{-} O R P H A N\left(A_{U}, B_{S}\right)$ is true if and only if $X_{U} \swarrow_{*} Y_{S}$.

Before proving this theorem, we establish two lemmas.

Lemma 1 Suppose $B_{S}$ rolls back $A_{U}$. If $A_{S} \in$ $U S R_{-} T O_{-} S Y S\left(A_{U}\right)$ then $A_{S} \preceq_{S} B_{S}$. If $C_{S}$ satisfies $B_{S} \preceq_{S} C_{S}$, then $A_{U} \swarrow_{U} S Y S_{-} T O_{-} U S R\left(C_{S}\right)$.

Proof Rolled-back states remain rolled-back, and we can only roll back states that have happened.

Lemma 2 Suppose user state interval $A_{U}$ and system state interval $B_{S}$ satisfy $A_{U} \longrightarrow S Y S_{-} T O_{-} U S R\left(B_{S}\right)$. Let $A_{S}$ be the minimal interval in USR_TO_SYS $\left(A_{U}\right)$. Then $A_{S} \Longrightarrow B_{S}$.

Proof We establish this result by induction: If $A_{U}$ and $S Y S_{-} T O_{-} U S R\left(B_{S}\right)$ occur at the same process, this is easily true. If $A_{U}$ sends a message that begins $S Y S_{-} T_{O_{-}} U S R\left(B_{S}\right)$, then some interval in $U S R_{-} T O_{-} S Y S\left(A_{U}\right)$ precedes $B_{S}$, so clearly $A_{S}$ must also. For more general precedence paths, choose an intermediate node $C_{U}$ with $A_{U} \longrightarrow C_{U} \longrightarrow$ SYS_TO_USR $\left(B_{S}\right)$, and choose the minimal $C_{S}$ from USR_TO_SYS $\left(C_{U}\right)$. Establish the result for $A_{U}$ and SYS_TO_USR $\left(C_{S}\right)$, and for $C_{U}$ and $S Y S_{-} T O_{-} U S R\left(B_{S}\right)$.

Proof (of Theorem 1) Suppose for user state interval $A_{U}$ at process $p$ and system state interval $B_{S}$ at process $q$, that KNOWABLE_ORPHAN $\left(A_{U}, B_{S}\right)$ holds. Then at some process $r$, there exists a user state interval $C_{U}$ and system state interval $D_{S}$ satisfying the statements: (1) $C_{U} \Longrightarrow A_{U}$, (2) $D_{S}$ rolls back $C_{U}$, and (3) $D_{S} \Longrightarrow B_{S}$. Statement (1) implies that $C_{U} \preceq_{U} X_{U}[r]$. Statement (2) and Lemma 1 imply that $C_{U} \npreceq_{U} S Y S_{-} T O_{-} U S R\left(E_{S}\right)$ for any $E_{S}$ satisfying $D_{S} \preceq_{S} E_{S}$. Statement (3) implies that $D_{S} \preceq_{S} Y_{S}[r]$. Hence $C_{U} \swarrow_{U} S Y S_{-} T O_{-} U S R\left(Y_{S}[r]\right)$, and thus $X_{U} \swarrow_{*} Y_{S}$.

Conversely, suppose $X_{U} \swarrow_{*} Y_{S}$. Then there exists a process $r$ with $X_{U}[r] k_{U} S Y S_{-} T O_{-} U S R\left(Y_{S}[r]\right)$. Let $C_{U}=X_{U}[r]$; let $C_{S}$ be the minimal state interval in USR_TO_SYS $\left(C_{U}\right)$. By the definition of a timestamp vector, $C_{U} \Longrightarrow A_{U}$. By Lemma 2 and hypothesis, $C_{S} \Longrightarrow B_{S}$, and thus $C_{S} \preceq_{S} Y_{S}[r]$. Since by hypothesis $C_{U} \swarrow_{U} S Y S_{-} T O_{-} U S R\left(Y_{S}[r]\right)$ a $D_{S}$ must exist such that $C_{S} \preceq_{S} D_{S} \preceq_{S} Y_{S}[r]$ and $D_{S}$ rolls back $C_{U}$. Consequently, $D_{S} \longrightarrow B_{S}$, and hence KNOWABLE_ORPHAN $\left(A_{U}, B_{S}\right)$.

\section{The Protocol}

Our protocol tests for orphans by comparing timestamp vectors across two levels of partial order time [26, 27]. Section 3.1 presents our protocol in terms of system and user state interval indices, functions to compare them, and functions to generate new indices. Section 3.2 presents some approaches to implementing these functions.

\subsection{Functions}

Each process maintains timestamp vectors $V_{S}$ and $V_{U}$ for the system and user computations, respectively. At process $p$, the $p$ entries of these vectors are its current state interval indices.

Sending Messages Figure 3 shows how messages are sent. To send a system-level message, a process $p$ sends its own name, the message text, and the timestamp vector of the current system state interval. To send a user-level message, a process $p$ sends a system-level message whose text contains two items: the user message text, and the timestamp vector of the current user state interval.

Receiving a System-level Message Figure 4 shows how processes receive system-level messages. ${ }^{2}$ Process $p$ first uses the timestamp vector on the message to update the system timestamp vector at $p$. Process $p$ then begins a new system state interval and asynchronously logs the timestamp vector of the new interval. (Only the maximum system timestamp vector from each process need be retained on stable storage.) The updated system timestamp vector gives process $p$ new information about the recovery activity of other processes. Process $p$ compares its current user timestamp vector to its new system timestamp vector to determine if this new information indicates that the user state at $p$ is now an orphan. If so, process $p$ calls ROLL_BACK to roll itself back to its most recent non-orphan user state interval. In any case, RECEIVE_ SYS at process $p$ returns the source of the system message and the message text.

$R E C E I V E_{-} S Y S$ uses the $N E W_{-} S Y S_{-} I N D E X$ function to generate new system state interval indices. $N E W_{-} S Y S_{-} I N D E X\left(A_{S}, B_{U}\right)$ returns a system state interval index that immediately follows $A_{S}$, and whose unique user state interval is $B_{U}$.

\footnotetext{
${ }^{2}$ The assumption of piecewise determinism requires user-level processes to perform blocking receives, and for clarity of presentation, we have also assumed that system-level processes perform blocking receives. However, we could obtain increased performance by having the system-level process perform interrupt-driven receives; the system process would maintain a buffer of messages for the user process, and on each $V_{S}$ update could re-examine the buffer for orphans.
} 


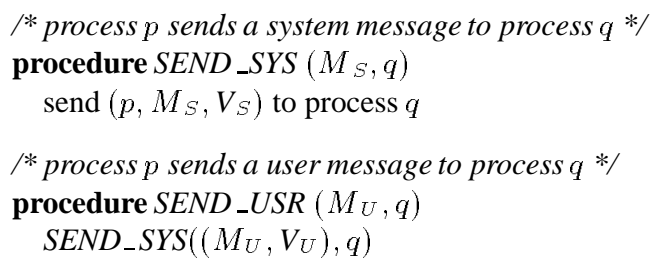

Figure 3 Sending system and user messages.

Rollback Figure 5 shows the $R O L L_{-} B A C K$ procedure, in which a process $p$ restores its most recent available user state interval that is not an orphan. If $p$ has failed and lost volatile storage, then the most recent user state intervals may not be available.

As with RECEIVE_ SYS, process $p$ determines orphans by comparing a user timestamp vector to the current system timestamp vector. Process $p$ first obtains its most recent checkpointed user state interval that is not an orphan. Process $p$ then replays its received messages logged after this checkpoint was recorded, until a message is reached whose send is an orphan. Process $p$ discards any orphan checkpoints and logged messages, and begins a new system state interval. The discarding of orphan log data must be completed before any new data is logged. ${ }^{3}$ ROLL_BACK also uses the NEW_INCARNATION function to generate new system state interval indices. The $N E W_{-} I N C A R N A T I O N\left(A_{S}, B_{U}\right)$ function returns a system state index $B_{S}$ whose user state is $B_{U}$. $B_{S}$ follows $A_{S}$ immediately - and also follows all other state intervals that have already occurred. (This is necessary since the process

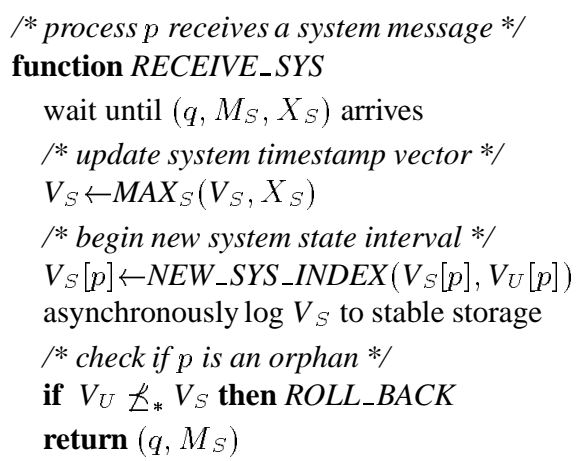

Figure 4 Receiving a system-level message.

\footnotetext{
${ }^{3}$ Old logged messages and checkpoints may also be discarded when no
} longer necessary for recovery from any possible future failure [13, 30].

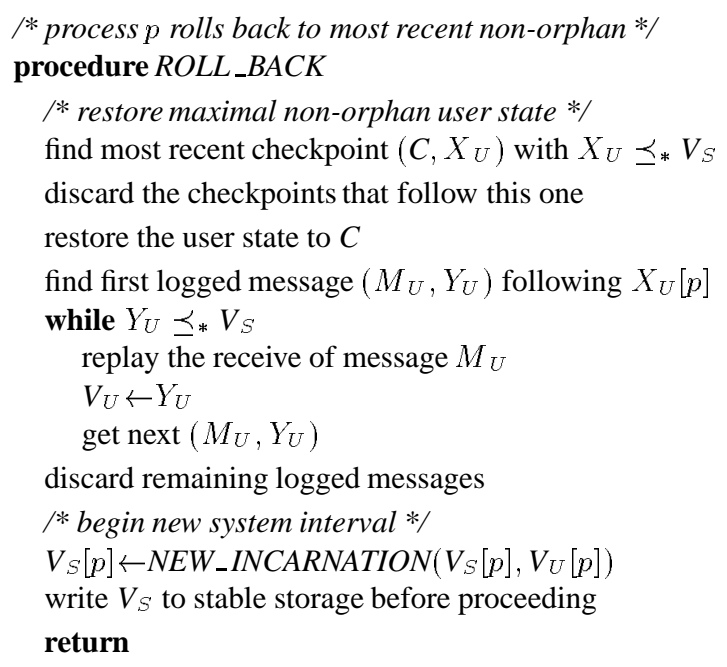

Figure 5 The rollback procedure for process $p$.

may have proceeded beyond $A_{S}$, but lost this information due to failure.)

Receiving a User-level Message Figure 6 shows how processes receive user-level messages. When receiving a user-level message, a process $p$ waits for a system-level message that carries a user-level message whose send is not an orphan (according to $p$ 's current information). Process $p$ then uses the user timestamp vector on the user message to update the user timestamp vector at $p .{ }^{4}$ Process $p$ then begins a new user state interval and a new system state interval.

RECEIVE_ $U S R$ uses the NEW_USR_INDEX function to generate new user state interval indices. The $N E W_{-} U S R_{-} I N D E X\left(A_{S}, B_{U}\right)$ function returns a user state interval index that immediately follows $B_{U}$, in the context of the system state interval index $A_{S}$.

Recovering from Failure For process $p$ to recover from its own failure, it simply reloads the current system timestamp vector from stable storage into $V_{S}$, and then calls ROLL_BACK.

How quickly the system recovers from process failure depends on how quickly the processes whose user state intervals are orphans (or will become orphans) learn of the failure. Our protocol allows a range of alternatives, from

\footnotetext{
${ }^{4}$ Theorem 1 establishes that this $M A X_{U}$ comparison is always well-defined. Suppose process $p$ were to accept a user message with user timestamp vector $X_{U}$, and for some $q, X_{U}[q]$ and $V_{U}[q]$ were incomparable under $\preceq_{U}$. Then either $X_{U}[q]$ had been rolled back by the time $V_{U}[q]$ occurred (so process $p$ would have discarded the message), or $V_{U}[q]$ had been rolled back by the time $X_{U}[q]$ had occurred (so process $p$ would have rolled itself back).
} 


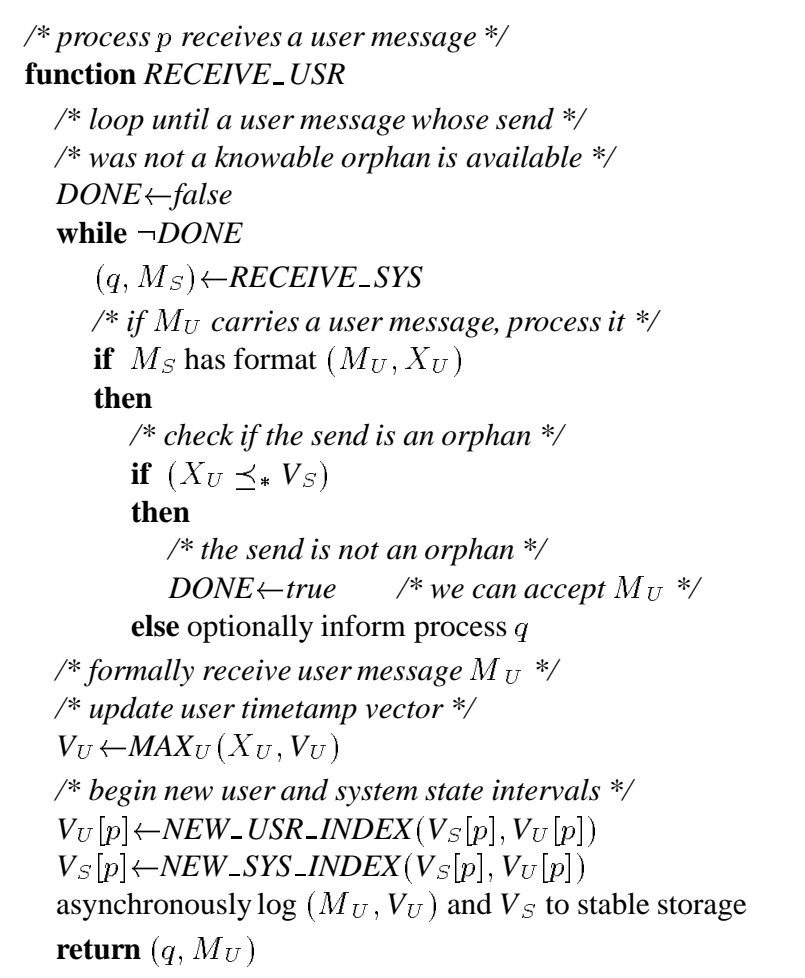

Figure 6 Receiving a user-level message.

unreliable broadcast of system-only messages, to percolation via the system timestamp data on user messages. Finding good heuristics here depends on the communications patterns of the computation.

An Example Figures 7 and 8 illustrate how our protocol avoids the multiple rollback problem of Figure 1.

\subsection{Implementation Considerations}

Our new protocol requires a way to represent user and system state indices, to compare these representations under the $\preceq_{U}, \preceq_{S}$ and $\preceq_{*}$ functions, and to generate new indices. This section provides one possible solution.

System Indices The system state interval indices should reflect the timeline structure of the system state intervals, but must also take into account the fact that a failed process may lose all state. The system index should also indicate the index of the corresponding user state interval. Following [30], we say that each rollback of a process begins a new incarnation of that process. We represent the index for system state interval $A_{S}$ as a triple $\left(k, m, A_{U}\right)$. Integer $k$ represents the incarnation in which $A_{S}$ occurs; integer $m$ represents the sequence of $A_{S}$ within that incarnation, and index $A_{U}$ indicates the user interval corresponding to $A_{S}$.

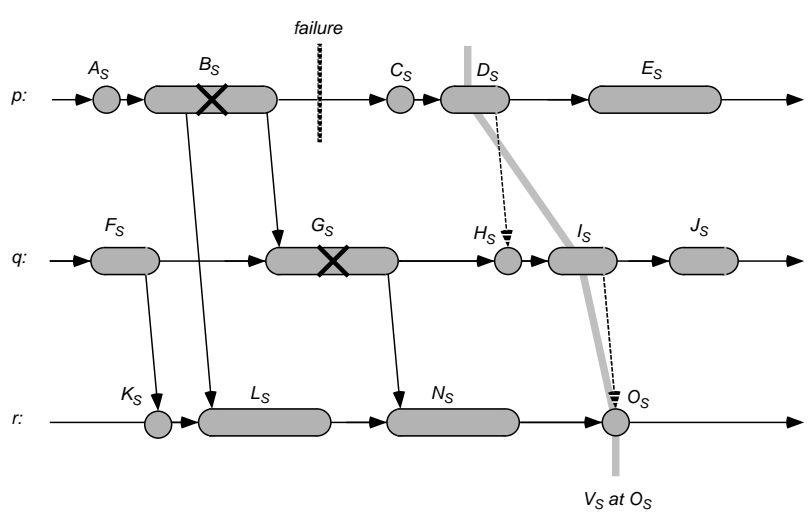

Figure 7 Our protocol avoids the multiple rollbacks that the Strom and Yemini protocol permits. This diagram shows the system partial order for a scenario like Figure 1. Here, process $p$ fails, rolls itself back, and announces this fact to process $q$, which rolls itself back and announces this fact to process $r$. (Dashed arrows indicate system-only messages).

This format supports our index generation functions. Let $A_{S}$ be a system state interval with index $\left(k, m, A_{U}\right)$. NEW_SYS_INDEX $\left(A_{S}, B_{U}\right)$ returns an index $\left(k, m+1, B_{U}\right)$. NEW_INCARNATION $\left(A_{S}, B_{U}\right)$ returns $\left(k^{\prime}, 0, B_{U}\right)$, where $k^{\prime}$ is one greater than the incarnation count at stable storage. This format also directly supports the $\preceq_{S}$ and $\preceq_{*}$ comparisons. For $\preceq_{S}$, we just lexico-

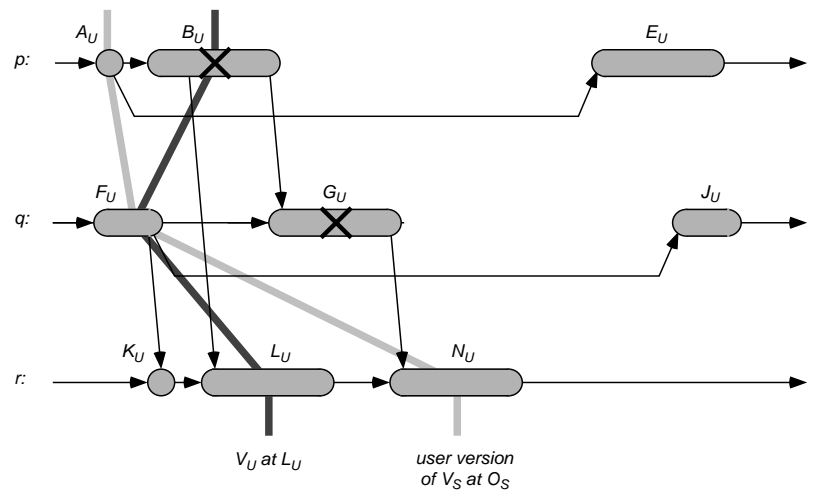

Figure 8 Unlike the Strom and Yemini protocol, our protocol causes process $r$ to roll back far enough the first time. This diagram shows the user partial order, and demonstrates how our orphan test achieves this result. The dark band shows the user timestamp vector of user state interval $L_{U}$; the light band shows the user version of the system timestamp vector of system state interval $O_{S}$. Since the dark band does not precede the light band at process $p$, process $r$ knows at $O_{S}$ that $L_{U}$ is an orphan. 
graphically compare the first two entries in the indices for two intervals. For $\preceq_{*}$, we extract the the third entry of the system index, and use $\preceq_{U}$ (defined below).

User Indices In order to reflect the timetree structure of the user state intervals, we can use a representation for user state interval indices that indicates the position of a user state interval within the timetree. With every user interval, we associate two integers: the depth of the interval within the timetree, and the system incarnation in which this interval first started. (The incarnation changes only when rollback occurs.) We represent the index of a user state interval $A_{U}$ as a triple $(i, j, S)$. Integer $i$ is the depth of $A_{U}$, integer $j$ is its incarnation, and set $S$ contains integer pairs indicating the depth and incarnation of all $B_{U}$ satisfying two conditions:

1. $B_{U} \preceq_{U} A_{U}$

2. Either $B_{U}$ has an incarnation different from its parent in the timetree, or $B_{U}$ the root of the timetree.

By specifying each branch, this set determines the path from the root of the timetree to $A_{U}$.

This index format provides straightforward support for the $\preceq_{U}$ comparison. Suppose user intervals $A_{U}$ and $B_{U}$ have indices $\left(i_{A}, j_{A}, S_{A}\right)$ and $\left(i_{B}, j_{B}, S_{B}\right)$, respectively. To evaluate whether $A_{U} \preceq_{U} B_{U}$, we determine first if $i_{A} \leq$ $i_{B}$, and then if $S_{A} \subseteq S_{B}$.

This index format also provides a straightforward way to generate new indices. Suppose system state interval $A_{S}$ has incarnation and sequence numbers $k$ and $m$, respectively; suppose user state interval $B_{U}$ has index $(i, j, S)$. If $k=j$, $N E W_{-} U S R_{-} I N D E X\left(A_{S}, B_{U}\right)$ returns $(i+1, k, S)$; if $k \neq j$, $N E W_{-} U S R_{-} I N D E X\left(A_{S}, B_{U}\right)$ returns $(i+1, k, S \cup\{(i+$ $1, k)\})$.

Reducing the Size of User Indices The size of the set in the index of a user interval $A_{U}$ is proportional to the number of rollbacks in the path from the root to $A_{U}$. If failures occur, this will not be constant; thus, for these indices, the size of user timestamp vectors will not be linear. Instead, the size will be proportional to the number of rollbacks in the system-past of the intervals recorded in the vector entries. (However, the number of vector entries will still be linear.)

We can reduce the amortized length of user indices by having processes avoid transmitting redundant data. One approach is to use a simple compression mechanism to abbreviate redundant byte sequences in the representation of timestamp vectors sent on a message. Another approach is to specify user index paths from intermediate nodes instead of from the root. Suppose process $p$ wants to send the index of $A_{U}$ to process $q$. Instead of sending the path from the root to $A_{U}$, process $p$ can send the path from an intermediate interval $B_{U}$ to $A_{U}$. If process $q$ already knows the path from the root to $B_{U}$, then process $q$ quickly reconstructs the full path. If not, process $q$ recognizes that it is missing data and can request it of process $p$.
One example of this amortization technique is using a heuristic similar to Strom and Yemini's approach. Each time a process rolls back, it broadcasts the path to that rollback node along with its new incarnation count. Subsequent user indices consist solely of the system incarnation count and the position of the user interval within that incarnation. (This heuristic introduces blocking into our protocol, but still maintains the at-most-once upper bound on rollbacks at a process.) However, a wide range of other heuristics exists for this technique. At one extreme, process $p$ transmits only the end of the path; at the other extreme, process $p$ maintains the most recent system timestamp vector received from $q$, and uses the $q$ entry as the intermediate node for a name sent to $q$. Commitment and garbage collection may integrate nicely with these amortization techniques, since processes may maintain a log vector of the maximal known logged nodes at other processes.

\section{Properties of the Protocol}

Our new protocol is the first optimistic rollback protocol to implement completely asynchronous recovery. We discuss the advantages.

Suppose a process $p$ fails and rolls itself back. A surviving process $q$ will roll back its own user state when this state fails the $\preceq_{*}$ comparison in RECEIVE_SYS. Hence:

- Complete Asynchrony When a process must roll back, it can roll back immediately and resume computation without additional synchronization with other processes.

Theorem 1 shows that surviving process $q$ will roll back its user state when this state becomes a knowable orphan: when it depends on a rolled back state, and a knowledge path exists from the rollback to $q$. Because of message logging, a surviving process can always restore its maximal non-orphan state, so the orphans created by a process failure are exactly the state intervals that depend on the computation lost at the failed process. Because processes test user messages before receiving them, the state at process $q$ never becomes an orphan due to the failure at $p$ once a knowledge path is established. Hence:

- Maximal Recovery Like other optimistic rollback protocols, ours guarantees that a state is rolled back if and only if it causally depends on the computation lost at failed processes.

- Minimal Rollbacks Our protocol also guarantees that a failure at process $p$ causes a process $q$ to roll back at most once. Processes that do not depend on the failure will not roll back at all.

- Speedy Recovery Suppose process $q$ must roll back because of a failure at process $p$. Process $q$ will roll back as soon as any knowledge path is established from $p$ 's rollback. 
- Toleration of Network Partitions Another sideeffect of our asynchronous approach is that once initiated, recovery can proceed despite a partitioned network. The only processes that need to worry about recovery are those that may causally depend on lost states. Since each such process can recover asynchronously, the processes on the same side of the partition as the failure can recover immediately. Processes on the other side that need to recover can do so when the network is reunited. The remaining processes on either side may proceed unhindered. (However, this paper does not address the problem of detecting failure in a partitioned network.)

The preceding discussion considered the failure of a single process. Using the $\preceq_{*}$ test allows a surviving process to roll itself back to the maximal state that is not an orphan due to any rollback within the survivor's knowledge horizon. Hence our protocol provides:

- Concurrent Recovery Recovery from a process failure occurs as information about the failure propagates. Basing recovery on information flow rather than coordinated rounds directly allows recovery from concurrent failures to proceed concurrently: the recoveries merge and the protocol restores the maximum recoverable system state. (In particular, two processes that each need to roll back due to two failures do not need to react to the failures in the same order.)

This protocol arose out of research into the security and privacy aspects of partial order time [28, 23]. Coupling this protocol with a fully secure implementation of partial order clocks [29] would yield a recovery protocol that tolerates not only faults, but also malicious acts of espionage and sabotage.

\section{Comparison to Related Work}

Strom and Yemini [30] initiated the area of optimistic rollback recovery. They presented optimistic techniques for surviving processes to ensure complete recoverability, and a rollback protocol ${ }^{5}$ that allows processes to recover mostly asynchronously, although delayed transmission of incarnation start information may cause blocking. Strom and Yemini's protocol uses an orphan test that is strictly weaker than ours. Their protocol never falsely concludes that a nonorphan state is an orphan. However, their protocol will falsely conclude that some orphan states are not orphans even when the testing process could potentially know otherwise. These false negatives make it possible for a single failure at one process to cause another process to roll back an

\footnotetext{
${ }^{5}$ In some sense, Merlin and Randell [19] foreshadowed Strom and Yemini's work by presenting a protocol based on a representation similar to Petri Nets; this protocol could be transformed and optimized into one similar to Strom and Yemini's.
}

exponential number of times, since the unfortunate process never rolls back far enough (until the last time).

Johnson and Zwaenepoel [12,13] developed a general model for optimistic rollback recovery. They used state lattices from partial order time to show that a maximal recoverable system state exists, and presented synchronized protocols to recover this state - even without reliable message delivery. Sistla and Welch [25] presented two protocols for optimistic recovery that avoid the exponential worst case by using synchronization between processes during recovery; like Strom and Yemini, Sistla and Welch require reliable FIFO message channels. Peterson and Kearns [20] recently presented a recovery protocol using vector clocks that synchronizes during recovery by passing tokens. However, we improve even on the explicit vector time work of Peterson and Kearns by truly using the full power of temporal abstraction.

Recovery protocols based on checkpointing without message logging restore the system to a recovery line composed of local checkpoints. Organizing recovery lines into an increasing sequence (e.g., [5,7]) may allow asynchronous recovery and may tolerate concurrent failures. However, unless for every state $A_{U}$, the maximal global state containing $A_{U}$ is a recovery line, checkpointing-based recovery will force surviving processes to roll back computation that does not depend on the computation lost due to failure

One approach to avoiding the exponential rollback of Strom and Yemini is to enforce that all messages are delivered in causal order, as in ISIS [2]. Another approach is to have each process piggyback all received failure messages on each outgoing message and thus restrict enforced causal delivery to failure announcements. Essentially, our protocol optimizes these approaches. The system timestamp vector concisely expresses the information processes would deduce via causal delivery of failure announcements. Focusing on this resulting state information permits arbitrary delivery orders.

\section{Conclusion}

Optimistic rollback protocols improve on other recovery methods by requiring little synchronization during failurefree operation and by requiring only the theoretical minimum amount of computation to be rolled back (since the only computation that must be rolled back is the computation that depends on the computation lost due to failure). Our protocol improves on previous optimistic rollback protocols by providing both completely asynchronous recovery and a worst-case upper bound of at most one rollback at each process. The key to asynchronous optimistic rollback recovery is the realization that two levels of partial order time abstraction are relevant: causal dependency on rolled-back 
events and potential knowledge of rollbacks. Our protocol explicitly tracks these two levels of time.

\section{References}

[1] B. Bhargava and S. Lian. "Independent Checkpointing and Concurrent Rollback Recovery for Distributed Systems An Optimistic Approach." Seventh Symposium on Reliable Distributed Systems. 3-12. IEEE, 1988.

[2] K. Birman, A. Schiper, and P. Stephenson. "Lightweight Causal and Atomic Group Multicast." ACM Transactions on Computer Systems. 9: 272-314. August 1991.

[3] A. Borg, J. Baumbach and S. Glazer. "A Message System Supporting Fault Tolerance." Ninth ACM Symposium on Operating Systems Principles. 90-99. 1983.

[4] A. Borg, W. Blau, W. Graetsch, F. Herrmann, and W. Oberle. "Fault Tolerance Under UNIX." ACM Transactions on Computer Systems. 7 (1): 1-24. February 1989.

[5] D. Briatico, A. Ciuffoletti, and L. Simoncini. "A Distributed Domino Effect Free Recovery Algorithm.” IEEE Symposium on Reliability in Distributed Software and Database Systems. 207-215. October 1984.

[6] K. M. Chandy and L. Lamport. "Distributed Snapshots: Determining Global States of Distributed Systems." ACM Transactions on Computer Systems. 3: 63-75. February 1985.

[7] A. Ciuffoelleti. "La Coordinazione Delle Attivita Di Ripristino Nei Sistemi Distribuiti." A.I.C.A. Annual Conference Proceedings. October 1989.

[8] E. N. Elnozahy, D. B. Johnson and W. Zwaenepoel. "The Performance of Consistent Checkpointing." Eleventh IEEE Symposium on Reliable Distributed Systems. 39-47. October 1992.

[9] E. N. Elnozahy and W. Zwaenepoel. "Manetho: Transparent Rollback-Recovery with Low Overhead, Limited Rollback and Fast Output Commit." IEEE Transactions on Computers. 41 (5): 526-531. May 1992

[10] C. J. Fidge. "Timestamps in Message-Passing Systems That Preserve the Partial Ordering." Eleventh Australian Computer Science Conference. 56-67. February 1988.

[11] D. B. Johnson and W. Zwaenepoel. "Sender-Based Message Logging." Seventeenth Annual International Symposium on Fault-Tolerant Computing. 14-19. 1987.

[12] D. B. Johnson. Distributed System Fault Tolerance Using Message Logging and Checkpointing. Ph.D. thesis, Rice University, 1989.

[13] D. B. Johnson and W. Zwaenepoel. "Recovery in Distributed Systems Using Optimistic Message Logging and Checkpointing." Journal of Algorithms. 11: 462-491. September 1990.

[14] D. B. Johnson. "Efficient Transparent Optimistic Rollback Recovery for Distributed Application Programs." Twelfth IEEE Symposium on Reliable Distributed Systems. 86-95. October 1993
[15] R. Koo and S. Toueg. "Checkpointing and RollbackRecovery for Distributed Systems." IEEE Transactions on Software Engineering. 13 (1): 23-31. January 1987.

[16] P. Leu and B. Bhargava. "Concurrent Robust Checkpointing and Recovery in Distributed Systems." Fourth International Conference on Data Engineering. 154-163. 1988.

[17] K. Li, J. F. Naughton and J. S. Plank. "Real-Time, Concurrent Checkpointing for Parallel Programs." Second ACM SIGPLAN Symposium on Principles and Practices of Parallel Programming. 79-88. 1990.

[18] F. Mattern. "Virtual Time and Global States of Distributed Systems." In Cosnard, et al, ed., Parallel and Distributed Algorithms. Amsterdam: North-Holland, 1989. 215-226.

[19] P. M. Merlin and B. Randell. "State Restoration in Distributed Systems." International Symposium on Fault-Tolerant Computing. June 1978.

[20] S. L. Peterson and P. Kearns. "Rollback Based on Vector Time." Twelfth IEEE Symposium on Reliable Distributed Systems. 68-77. October 1993.

[21] M. L. Powell and D. L. Presotto. "Publishing: A Reliable Broadcast Communication Mechanism." Ninth ACM Symposium on Operating Systems Principles. 100-109. 1983.

[22] B. Randell. "System Structure for Fault Tolerance." IEEE Transactions on Software Engineering. SE-1: 220-232, 1975.

[23] M. Reiter and L. Gong. "Preventing Denial and Forgery of Causal Relationships in Distributed Systems." IEEE Symposium on Research in Security and Privacy. 1993.

[24] D. L. Russell. "State Restoration in Systems of Communicating Processes." IEEE Transactions on Software Engineering. 6 (2): 183-194. March 1980.

[25] A. P. Sistla and J. L. Welch. "Efficient Distributed Recovery Using Message Logging." Eighth ACM Symposium on Principles of Distributed Computing, 223-238. August 1989.

[26] S. W. Smith. A Theory of Distributed Time. Computer Science Technical Report CMU-CS-93-231, Carnegie Mellon University. December 1993.

[27] S. W. Smith. Secure Distributed Time for Secure Distributed Protocols. Ph.D. thesis. Computer Science Technical Report CMU-CS-94-177, Carnegie Mellon University. September 1994.

[28] S. W. Smith and J. D. Tygar. Signed Vector Timestamps: A Secure Protocol for Partial Order Time. Computer Science Technical Report CMU-CS-93-116, Carnegie Mellon University. October 1991; version of February 1993.

[29] S. W. Smith and J. D. Tygar. "Security and Privacy for Partial Order Time." Seventh ISCA International Conference on Parallel and Distributed Computing Systems. October 1994.

[30] R. Strom and S. Yemini. "Optimistic Recovery in Distributed Systems." ACM Transactions on Computer Systems. 3: 204226. August 1985.

[31] Y.-M. Wang and W. K. Fuchs. "Lazy Checkpoint Coordination for Bounding Rollback Propagation." Twelfth IEEE Symposium on Reliable Distributed Systems. 78-85. October 1993. 